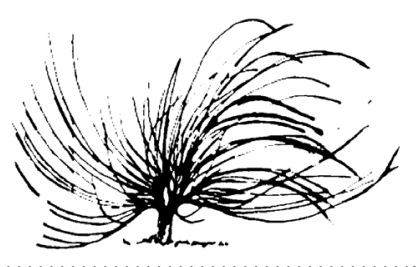

\title{
Educación como derecho humano, perspectiva desde la educación matemática costarricense
}

\author{
Marianela Zumbado-Castro ${ }^{1}$ \\ Universidad Estatal a Distancia \\ Costa Rica \\ mazumbado@uned.ac.cr
}

\begin{abstract}
Resumen
La educación como derecho debe facilitar a todas las personas la posibilidad de aprender a aprender $\mathrm{y}$ hacerlo a través de toda su vida; además, debe poseer cuatro características para ser considera como derecho: disponibilidad, accesibilidad, aceptabilidad y adaptabilidad. Por tanto, se pretende a través de la presencia de estas cuatro características, permitir a los estudiantes ser aprendices expertos. En este proceso contribuye en Costa Rica, la política educativa, la curricular y los programas de ciencias y matemáticas, que promueven la indagación, las diferentes maneras de pensar y la resolución de problemas. Por lo tanto, el propósito de este trabajo es analizar el aporte de la educación matemática costarricense a la consolidación de la educación como derecho humano, a través de la formación de aprendices expertos y sus limitaciones. Dos resultados de esta reflexión son: que la implementación de los programas de matemáticas, sin la aplicación de la fundamentación teórica, constituye una limitación
\end{abstract}

Recibido: 6 de mayo de 2019. Aprobado: 12 de setiembre de 2019.

http://dx.doi.org/10.15359/rep.14-2.6

1 Tutora de la Cátedra de Didáctica de la Matemática e integrante del Proyecto Reforma de la Educación Matemática en Costa Rica, adscrito al Ministerio de Educación Pública de Costa Rica. Magíster en Educación, Universidad Latina de Costa Rica. ORCID: https://orcid. org/0000-0002-5774-1884 
para los aprendices expertos y el otro, que es necesario supervisar la ejecución de las ideas plasmadas en las mallas curriculares, para garantizar el derecho de aprender para la vida.

Palabras clave: derecho a la educación, oportunidades educativas, educación fundamental, alfabetización funcional, Enseñanza de las Matemáticas.

\begin{abstract}
Education as a right should enable all people to learn to learn and to do so throughout their lives; also, it must have four characteristics to be considered a right: availability, accessibility, acceptability, and adaptability. Therefore, through the presence of these four characteristics, it is intended to allow students to be "expert learners". In this process, Costa Rica contributes educational policy, curricular policy, and science and mathematics programs that promote inquiry, different ways of thinking, and solving problems. Therefore, the purpose of this paper is to analyze the contribution of Costa Rican mathematics education to the consolidation of education as a human right through the training of expert apprentices and their limitations. Two results of this reflection are that the implementation of the mathematics programs, without the application of a theoretical foundation constitutes a limitation for the expert learners and that it is necessary to supervise the execution of the ideas expressed in the curriculum for guaranteeing the right to learn for life.
\end{abstract}

Keywords: Human rights, educational opportunities, fundamental education, functional literacy, mathematics education

\title{
Introducción
}

a Declaración Universal de Derechos Humanos (ONU, 1948), establece en su Artículo 26, que la educación es un derecho y con esto se marca el inicio de profundas reflexiones sobre el 
ser, su lugar, sus posibilidades, así como sus necesidades y condiciones mínimas para su desarrollo.

En 1999, la Organización de Naciones Unidas (ONU) establece que la educación debe poseer cuatro características esenciales para poder ser considerada como un derecho y son las siguientes: disponibilidad, accesibilidad, aceptabilidad y adaptabilidad, las cuales deben estar relacionadas estrechamente y estar presentes en los entornos educativos.

La disponibilidad, implica una oferta diversa que satisfaga las necesidades de la sociedad, la atención de niños, adolescentes, adultos y adultos mayores. Además, ofertas de diversos tipos: académicas, ocupacionales o técnicas, de alfabetización, formación continua, entre muchas otras. Asimismo, se debe poseer una infraestructura con diseño universal, con baterías sanitarias, agua potable, iluminación y mobiliario. También, servicios de biblioteca con acceso a tecnología y profesionales capacitados (ONU, 1999; UNESCO, 2015). Todos estos componentes conforman la noción de disponibilidad en educación.

Respecto a la accesibilidad, para que la educación cumpla con esta característica debe ser inclusiva y con un diseño universal (MEP, 2018b), sin tolerancia a la discriminación, gratuita con un alcance hasta educación secundaria y terciaria, con asequibilidad de materiales físicos o digitales (ONU, 1999; UNESCO, 2015; Riveros, 2017).

La aceptabilidad implica "los programas de estudio y los métodos pedagógicos" (ONU, 1999, p. 3), esta característica también incluye dos ideas, la primera sobre la calidad en las ofertas educativas y la segunda sobre la pertinencia cultural para la comunidad (Muñoz, 2013; UNESCO, 2015).

La cuarta característica es la adaptabilidad, el concepto que está involucrado es "flexibilidad", la educación se debe adaptar a las comunidades y a sus necesidades. Según Muñoz (2013): "tiene como base el reconocimiento de la diversidad humana y busca que los establecimientos educativos desarrollen procesos curriculares y administrativos con la flexibilidad suficiente que les permita adaptarse a las necesidades de las y los estudiantes" (p. 55).

Se ha mencionado que estas cuatro características deben estar relacionadas de manera estrecha e indivisible, esto es evidente, debido a que la educación debe ser para todos, por tanto, debe estar a disposición de cada individuo, según sus características personales: edad, sexo, preferencias sexuales, particularidades físicas, creencias religiosas, así como 
las condiciones sociales, económicas, culturales y migratorias, entre muchas otras (Limas y Ampudia, 2013; UNESCO, 2015; Riveros, 2017).

Otro aspecto importante es que la educación debe trascender el acceso a la escolarización, ir hacia el desarrollo de habilidades para la vida a través del perfeccionamiento personal y colectivo (Muñoz, 2013). Asimismo, se debe pretender que los estudiantes se transformen en "aprendices expertos" (Cast, 2008, según se cita en MEP, 2018b, p. 5), donde los contenidos no son lo más importante, sino las habilidades para aprender, aprender a aprender y aprender a utilizar lo aprendido ( $\sin$ importar que esto sea un contenido disciplinar, una habilidad o destreza, un valor o una actitud); que tengan la capacidad de establecer estrategias para capturar algún conocimiento de su interés o por alguna necesidad de la vida cotidiana. Además, deben poder discriminar sobre los recursos didácticos o tecnológicos que sean de mayor utilidad y autoincentivar la perseverancia para lograr metas y continuar aprendiendo toda la vida (Torres, 2005; MEP, 2018; Ossa-Cornejo, Palma-Luengo, Lagos-San Martín y Díaz- Larenas, 2018).

Algunas preguntas que surgen de estas ideas son ¿cómo lograr que una educación que debe cumplir cuatro características proporcione condiciones para desarrollar aprendices expertos? ¿Qué propone el Ministerio de Educación Pública (MEP) costarricense para lograr aprendices expertos? ¿Cuál es el papel de la educación matemática para favorecer a esos aprendices?, a raíz de estos cuestionamientos surge el objetivo general de este ensayo, analizar el aporte de la educación matemática costarricense a la consolidación de la educación como derecho humano, a través de la formación de aprendices expertos y sus limitaciones.

Según Torres (2005), se debe promover el aprendizaje "a lo largo y a lo ancho de la vida" (p. 80), esto significa que desde el nacimiento hasta la muerte se debe adquirir conocimiento, pero no solamente a través del tiempo, sino mediante múltiples espacios, donde la escuela es solamente un lugar. Por tanto, se debe desarrollar en los estudiantes el hábito de preguntar, para esto es necesario hacer cultural los procesos asociados a la ciencia (curiosidad, indagación, sistematización, comunicación) y el análisis crítico (Ossa-Cornejo et al., 2018).

$\mathrm{Al}$ respecto, Tamayo y Orrego (2005), plantean que las propuestas curriculares deben llevarnos a "que se reflexione acerca de las relaciones entre la ciencia y su conocimiento público, acerca del logro de una mejor comprensión pública de la ciencia y del desarrollo de habilidades 
para la toma de decisiones relacionadas con problemas socio-científicos" (p. 14). Esto implica hacer parte de la cultura costarricense el pensamiento científico, las aulas deben ser espacios para aprender ¿qué es un objeto en su entorno? mediante el trabajo colectivo, lo que simulará una comunidad científica mientras se resuelven problemas del contexto, como lo plantea la educación matemática y en particular los programas oficiales del MEP (2012), como se verá posteriormente.

De esta manera se lograrán desarrollar habilidades en los estudiantes para que se conviertan en aprendices durante toda su vida. Al propiciar experiencias que permitan la indagación de manera constante en los entornos educativos, se convertirá en un estilo de vida (Ossa-Cornejo et al., 2018). Se debe indicar que, si se asume una cultura de pensamiento científico se pueden operacionalizar las cuatro características que debe tener la educación para ser un derecho humano, debido a que se promueve el trabajo en equipo, donde se asumen las diferencias personales, de criterio, de conocimiento y de cultura, como recursos para enfrentar problemas y con ello, se combate la discriminación y se asume la diversidad. También, se logra la adaptabilidad y la aceptabilidad de un modo natural, a través del enfrentamiento con problemas del contexto.

Respecto al pensamiento científico, Costa Rica no se ha quedado rezagada, desde el MEP se asume un compromiso de generar aprendices expertos desde su política curricular y educativa, así como algunos de sus programas de estudio, en particular los de ciencias y matemáticas (MEP, 2012, 2015, 2017a, 2017c, 2018b).

\section{Política educativa, política curricular y programas de estudio}

Para poder comprender cuál es la contribución de la educación matemática a los aprendices expertos, es necesario revisar el contexto político de manera preliminar, debido a que en él se enmarcan los programas oficiales, por lo tanto, se establecerán las ideas básicas de la política educativa como el marco general, la política curricular como la delimitación más próxima al estudiante y finalmente, el detalle a través de los programas específicos de ciencias y matemáticas, con énfasis en estos últimos.

\section{Política educativa}

En 2017, fue aprobada la política educativa denominada " $\mathrm{La}$ persona: centro del proceso educativo y sujeto transformador de la 
sociedad" (MEP, 2017a), cuyo marco filosófico y conceptual contiene el paradigma de la complejidad, el humanismo, el constructivismo social y racionalismo.

El MEP (2017a), manifiesta que se "fortalecerán el desarrollo del pensamiento científico, a partir de la indagación, la investigación y la experimentación" (p. 13). Asimismo, establece que los docentes deben implementar estrategias educativas para estimular "la curiosidad intelectual, la indagación, el razonamiento riguroso y la cultura científica" (p. 19).

Desde la complejidad, el MEP (2017a) asume el aprendizaje de cada persona como un conjunto bioecosocial, que le debe permitir aprender y tener la capacidad de crear e integrar sus ideas con el entorno. El humanismo aporta la visión autoevaluadora del sujeto como individuo sobre su aprendizaje, con la potencialidad de solucionar problemas. Desde el constructivismo social se extrae la importancia del contexto, las experiencias previas, las estructuras mentales preconstruidas y las raíces culturales, con lo anterior se enfatiza el conocimiento producto de contacto social. Finalmente, el racionalismo incorpora al marco filosófico y conceptual, la importancia de seleccionar conocimientos válidos para el momento histórico y social, esto último orienta la selección de contenidos para las mallas curriculares.

Asimismo, la política educativa asume dos ideas más. La primera de ellas, el concepto de calidad como la capacidad de centrar la atención en el estudiante, para poder propiciar el desarrollo de competencias, para "la comprensión, expresión e interpretación de conceptos, pensamientos, sentimientos, hechos y opiniones" (MEP, 2017a, p. 12). La segunda, la educación como un proceso continuo y para esto propone el desarrollo de "valores y actitudes, habilidades y competencias, que les permitan aprender a lo largo de la vida, por medio del autoconocimiento, la toma de acciones, decisiones y la construcción activa y proactiva de la ciudadanía" (p. 13). Además, es importante destacar que se enfatiza el papel de la familia para lograr el crecimiento que implican estas acciones.

\section{Política curricular}

En 2016, fue aprobada la política curricular denominada: "Educar para una nueva ciudadanía" (MEP, 2015), las habilidades perseguidas están plasmadas en cuatro dimensiones denominadas: maneras de pensar, formas de vivir en el mundo, formas de relacionarse con otros y 
herramientas para integrarse al mundo, las cuales poseen indicadores que las delimitan.

Respecto a la primera, maneras de pensar, se hace énfasis en el desarrollo cognitivo y se asocia con la generación de conocimiento, a través del uso de los pensamientos sistémico y crítico, las habilidades para aprender a aprender, la resolución de problemas, la creatividad y la innovación (MEP, 2015).

Las otras tres, se asocian con el "saber ser", respecto a formas de vivir en el mundo, se relacionan con el desarrollo e interrelación entre las culturas, la responsabilidad social y un proyecto de vida exitoso que considere la salud y el área profesional. Al establecer la dimensión formas de relacionarse con otros, se visualizan habilidades para la comunicación y trabajo en equipo. La última dimensión, herramientas para integrarse al mundo, se vincula con el desarrollo de capacidades para el manejo de tecnologías digitales e información.

Por tanto, el MEP establece en estas dimensiones las condiciones ideales para favorecer lo que se ha definido como aprendices expertos. Pero esto no está limitado a la política educativa y curricular, sino que está explícito en los programas de estudio.

\section{Programas de estudio}

Dos ejemplos concretos son los programas de ciencias y matemáticas. Respecto a los primeros, se propone el abordaje de la realidad a través del planteamiento de preguntas (indagación), esto implica la reflexión, la recolección de evidencias, la ejecución de pruebas, la contrastación de hallazgos y explicaciones, para luego continuar hallando respuestas. Asimismo, se promueve la rigurosidad, la honestidad, la objetividad y la refutabilidad a través de argumentos (MEP, 2017b, 2017c, 2017d, 2017e, 2017f).

Además, se establece una estrategia metodológica basada en indagación y se propone un "ciclo de mediación basado en la indagación" (MEP, 2017c, p. 18), el cual implica un papel activo de los estudiantes, pasando por diferentes momentos en el proceso de construcción de los conocimientos, que se produce al tratar de resolver algún problema, desafío o situación del contexto que despertó el interés, estos momentos son: la focalización, la exploración, la reflexión, la contrastación y la aplicación (MEP, 2017c). 
Los programas de matemáticas por su parte, establecen la resolución de problemas como estrategia metodológica principal, la cual a través de cuatro momentos predefinidos, permite a los estudiantes enfrentar situaciones contextualizadas o abstractas que representan un reto cognitivo, que implica emplear los conocimientos previos, plantear y ejecutar una estrategia de solución y validarla durante el momento de la "discusión interactiva y comunicativa" y el "cierre o clausura de la clase", aunque este último momento es una responsabilidad directa del docente (MEP, 2012, p. 41).

Estos programas pretenden desarrollar habilidades vinculadas con conocimientos matemáticos, pero a su vez, capacidades cognitivas superiores, que se construyen a través de los cinco procesos matemáticos (Ruiz, 2018), entendidos como razonar y argumentar, plantear y resolver problemas, comunicar y representar las ideas matemáticas, así como establecer conexiones con otras áreas del conocimiento (MEP, 2012).

Ciencias y matemáticas para el MEP, son dos asignaturas que responden a la necesidad de crear una cultura de pensamiento científico que favorecen el desarrollo de las personas como aprendices expertos, debido a que se promueve la pregunta, el problema o el reto como fuente de motivación para saber, averiguar, hallar o establecer una posible respuesta.

Es importante señalar, cómo las ideas de la política educativa y las dimensiones que orientan la política curricular están plasmadas en los programas, hasta permear la propuesta del planeamiento, para la organización de las lecciones: para ciencias, momentos denominados focalización, exploración, reflexión, contrastación y aplicación; para matemáticas, planteo de un problema, trabajo estudiantil independiente, discusión interactiva y comunicativa, finalmente, cierre o clausura. Para ambos planteamientos, existe una exposición de los estudiantes ante una situación retadora, el trabajo cognitivo de los alumnos sobre el objeto de interés, una socialización y un momento final que invita al contacto formal con los conocimientos involucrados, por tanto, se promueve que los alumnos asuman un rol activo (Ruiz, 2014).

A través de los programas de ciencias y matemáticas, con sus respectivas estrategias metodológicas: basada en indagación y resolución de problemas, es posible visualizar de manera transversal ambas políticas, cuando se permite a los estudiantes enfrentar un reto científico o matemático, se propician espacios para activar las diferentes maneras de pensar e implica que las habilidades para "saber ser" encuentran las 
condiciones ideales para ser activadas. Por tanto, el marco filosófico conceptual es operacionalizado, debido a que la complejidad de las situaciones de contexto hace surgir los elementos detallados por el MEP (2017a), sobre el humanismo, el constructivismo social y racionalismo, porque no es posible individualizarlas durante una indagación o la resolución de un problema contextualizado.

\section{Contribución de la educación matemática a los aprendices expertos}

Con base en las ideas anteriores, se puede establecer que los programas de matemáticas del MEP (2012), pueden contribuir al desarrollo de aprendices expertos, desde la visión de la educación matemática costarricense, plasmada en la malla curricular.

Se ha establecido que la educación será un derecho si se logra que las personas aprendan a aprender y a usar los conocimientos adquiridos para la vida. Entonces, si con los programas de matemáticas se persigue la siguiente competencia matemática:

(...) una capacidad del individuo para formular, emplear e interpretar las Matemáticas en una variedad de contextos. Incluye razonar matemáticamente y usar conceptos, procedimientos, hechos y herramientas para describir, explicar y predecir fenómenos. Ayuda a los individuos a reconocer el papel de las Matemáticas en el mundo y hacer juicios bien fundados y decisiones necesarias para ciudadanos constructivos, comprometidos y reflexivos. (OECD, 2010, p. 4, según se cita en MEP, 2012, p. 23)

Se puede concluir que la malla curricular favorecerá a los aprendices expertos, debido a que persigue capacidades necesarias para la vida, de manera que la toma de decisiones siempre sea fundamentada y esto contribuye a la noción de la educación como derecho, porque se otorga a la persona una herramienta para decidir (Callejos, 2000; Ruiz, 2014). Ahora bien, cómo contribuye la visión del área de números, medidas, geometría, relaciones y álgebra, también estadística y probabilidad, para formar personas con la capacidad de seguir aprendiendo durante toda la vida y con la posibilidad de mejorarla gracias a los conocimientos adquiridos en matemáticas en cualquier espacio de aprendizaje a lo ancho de la vida (NCTM, 2000; Torres, 2005), a continuación, se describen algunas contribuciones por área. 


\section{Números y medidas}

Según el MEP (2012), la primera área matemática citada aporta la posibilidad de desarrollar habilidades como identificar, leer, comprender y utilizar las diferentes representaciones de los números, el cálculo de operaciones básicas con enteros y racionales entre otros, cálculo mental y estimación. Unido a estas destrezas están aquellas que se propician a través del área denominada Medidas, en esta se plantea la necesidad de medir las características físicas de los objetos, como el área, el volumen y la capacidad, así como el uso correcto de las unidades de medida entre ellas: tiempo y distancia, también se incluye la conversión de la moneda. Estas son destrezas para la vida que se pretenden desarrollar desde el primer grado y fortalecer hasta el quinto año, se debe señalar que el área de Números se aborda en secundaria hasta el sétimo año de manera explícita y Medidas se trabaja de manera transversal hasta finalizar la secundaria.

De acuerdo con Calvo, Deulofeu, Jareño y Morera (2016), es de suma importancia conseguir una evolución en el sentido numérico y la capacidad de cálculo, debido a que se obtienen destrezas para la comprensión de los significados y características de los tipos de números, así como su uso pertinente de acuerdo al contexto, esto tiene como consecuencia la pericia para realizar cálculos básicos, determinar su veracidad y decidir entre la exactitud y la aproximación de acuerdo a la realidad que se enfrenta.

\section{Geometría}

Respecto a la contribución de esta área, ella aporta la visualización espacial que está estrechamente ligada con medidas y el cálculo de la capacidad de algunos cuerpos geométricos como las cajas y los envases (MEP, 2012), destrezas necesarias para la vida diaria, por ejemplo, para tomar decisiones de la veracidad de las ofertas sobre bebidas gaseosas en un supermercado. En los niveles avanzados se pueden favorecer habilidades para incursionar en disciplinas como la arquitectura, el diseño gráfico o carreras que requieren habilidades con el manejo del espacio o de la geometría del espacio.

Es importante señalar que, según la OCDE (2017), la visualización espacial, pertenece al área de conocimiento denominado Espacio y forma la cual incluye situaciones visuales y físicas del entorno, esto implica "patrones, propiedades de los objetos, posiciones y direcciones, 
representaciones de los objetos, descodificación y codificación de información visual, navegación e interacción dinámica con formas reales, así como con representaciones" (p. 75), por tanto, trasciende los contenidos tradicionales de la Geometría, porque implica la interacción con las medidas y el álgebra, favoreciendo las condiciones para aumentar las destrezas en los estudiantes.

\section{Relaciones y álgebra}

Esta área contribuye al desarrollo de capacidades desde la primera infancia, sobre las relaciones (pensamiento algebraico), concepto necesario para manejar apropiadamente el dinero, las aproximaciones del volumen de un cuerpo o la tarifa del taxi. Conforme se avanza en los años escolares, propicia habilidades de abstracción, representación o la modelación de situaciones del entorno (MEP, 2012). Se puede destacar aquí como la capacidad de identificar una relación o un patrón puede ayudar a los aprendices expertos a establecer que existe un problema o de manera complementaria, a la construcción de una estrategia de solución.

Es conveniente ofrecer a los estudiantes una visión apropiada de esta área matemática, el manejo algebraico sin valor práctico, implica la omisión de su utilidad como lenguaje y como herramienta para determinar relaciones numéricas o leyes que permitan la generalización de un evento; es más importante generar en los aprendices expertos destrezas para observar, describir, establecer relaciones y tratar de representarlas, que promover algoritmos para la resolución de ecuaciones (Calvo et al., 2016).

\section{Estadística y probabilidad}

Por su parte, esta área tiene un propósito fundamental, alfabetizar la población estudiantil sobre los datos y la casualidad (MEP, 2012). Según el ente ministerial, el trabajo en esta área, se orienta "por un lado a la identificación, organización y presentación de la información, lo que se asocia a la estadística descriptiva y por el otro la probabilidad que refiere al estudio de la incertidumbre y el azar" (MEP, 2012, p. 22), por tanto, su contribución es aumentar las herramientas de los estudiantes para el momento en que deben tomar decisiones y como se ha indicado previamente, esto aporta a la formación de los aprendices expertos.

La cultura estadística viene a ofrecer facilidades para interpretar los fenómenos científicos y sociales, elemento indispensable en 
la cultura de pensamiento científico que corresponde a un fin para el MEP. Estas áreas ofrecen la posibilidad de cuestionar la validez de la información estadística y analizar las probabilidades de un evento (confiabilidad), desde una postura crítica, con argumentos sólidos y con la capacidad de comunicar sus ideas correctamente, sin dejar de lado la formación que se requiere para la recolección de datos y la presentación de los resultados, esto involucra de manera directa los procesos matemáticos que se vinculan con capacidades cognitivas superiores (Calvo et al., 2016, Chaves, 2016; Ruiz, 2018).

A modo de síntesis, se puede establecer que la educación matemática, puede ofrecer a los aprendices expertos, oportunidades para aprender a aprender sobre contenidos de utilidad para la vida, porque se centra en conocimientos de un currículo que se puede relacionar con el contexto (Ruiz, 2014), lo que permite poner las cuatro características señaladas de la educación en práctica, debido a que la resolución de problemas, que es la estrategia metodológica principal propuesta por el MEP (2012), permite su aplicación a cualquier tipo de población, se puede plantear un problema contextualizado para niños o adultos mayores, sobre aritmética, finanzas o interpretación de información de un periódico y mostrarlo a través de audio, video, material concreto o texto (Bosman y Schulze, 2018) y enfrentarlo a través de trabajo grupal. Por tanto, el abordaje de las matemáticas mediante la propuesta del MEP (2012), permite propiciar la disponibilidad, accesibilidad, aceptabilidad y adaptabilidad de manera integral, operacionalizando la educación como derecho humano.

\section{Limitaciones para la formación de aprendices expertos}

Frente al potencial que ofrece la propuesta curricular de matemáticas de concretizar la educación como derecho humano que satisface las cuatro características mencionadas, está la realidad de las aulas.

Para garantizar aprendices expertos, se deben poseer condiciones que garanticen el éxito de la implementación de los programas, esto irremediablemente implica asuntos administrativos, de infraestructura y curriculares.

En este texto, se centrará la atención en los aspectos curriculares, en particular la mediación pedagógica, que se relaciona directamente y en primera instancia con la característica denominada aceptabilidad. En diversas investigaciones se ha señalado que en las aulas de secundaria 
no se evidencia la organización de las lecciones, según la propuesta del MEP (2012), que aún cuando los docentes admiten las ventajas de implementar la resolución de problemas como estrategia metodológica, no en todos los centros educativos del país están exponiendo a los estudiantes a entornos que propicien las destrezas para convertirse en aprendices expertos (Ruiz, 2013 y 2014; Ruiz y Barrantes, 2015; PEN, 2017; Meza, Agüero y Suárez, 2019).

Algunos docentes, no tienen dominio de los programas oficiales, en ciertas regionales educativas, los procesos de actualización y capacitación sobre la temática no fueron implementados de acuerdo con las expectativas del ente empleador, por diversas razones: zonas vastas de cobertura, docentes con deficiencias en formación inicial, ausencia del dominio de las tecnologías digitales, obstáculos en la gestión de permisos, viáticos y espacios para realizar las sesiones presenciales de capacitación, entre otras. Por tanto, existe un grupo significativo de educadores que no están aplicando los programas de matemáticas de acuerdo con su fundamentación teórica y esto obstaculiza el derecho a la educación (Ruiz, 2013; Ruiz y Barrantes, 2015; PEN, 2017; MEP, 2018a; Meza, Agüero y Suárez, 2019).

Lo anterior, implica que en las aulas hay ausencia de situaciones, tareas matemáticas o problemas retadores con diferentes niveles de complejidad durante la mediación pedagógica y esto limita la presencia de los procesos matemáticos, que se asocian con la generación de capacidades cognitivas superiores (Ruiz, 2018), y elementos teóricos incluidos en los fundamentos de los programas (MEP, 2012).

Asimismo, el desconocimiento de los programas oficiales implica que el estilo para la organización de las lecciones en dos etapas y cuatro momentos, que favorecerían cada una de las cuatro características ya mencionadas (disponibilidad, accesibilidad, aceptabilidad y adaptabilidad) no está siendo realidad en las instituciones educativas.

\section{Conclusiones}

Desde 1948, fue declarada la educación como derecho humano, sin embargo, aún en la actualidad, existen muchos obstáculos para que todas las personas gocen de este. Es importante señalar que aquellas sociedades, donde se han dado pasos en la dirección correcta, como en la costarricense, es necesario visualizar cómo se puede optimizar ese avance. 
Cambiar un paradigma empleando únicamente el discurso como herramienta no es posible, no se pueden desarrollar capacidades científicas si se siguen empleando las prácticas educativas anteriores, donde los estudiantes tenían un rol pasivo, el cambio será efectivo hasta que las propuestas del MEP para matemáticas y ciencias de los años 2012 y 2017, sean ejecutadas fielmente.

Es por eso, que se pretende evidenciar cómo la perspectiva de la educación matemática que está inmersa en la malla curricular costarricense, enmarcada en su política educativa y curricular, ofrece recursos concretos para favorecer una cultura nacional más científica que permita a las personas convertirse en aprendices expertos, por ejemplo, las habilidades en aritmética y la cultura estadística son dos herramientas que a cualquier ciudadano que desee investigar le serán de utilidad para enfrentar las preguntas que le genere su incesante necesidad de indagar.

Es importante señalar que la implementación de los programas de matemáticas, sin la aplicación de la fundamentación teórica, constituye una limitación para los aprendices expertos, debido a que se limitan las posibilidades de generar capacidades cognitivas superiores (Ruiz, 2018), si las lecciones de matemáticas no se trasforman en espacios para generar una cultura de pensamiento científico, las cuatro características del derecho a la educación no se cumplen y este derecho se violentan (Ossa-Cornejo et al., 2018).

Una educación matemática de acuerdo con las ideas del MEP (2012), puede ofrecer una educación deseable, sus alcances pueden superar las expectativas de los retractores, están diseñados con un nivel de generalidad que permite formar ciudadanos que hagan valer sus derechos y los de sus semejantes (Callejos, 2000).

En el nivel nacional, aun cuando al menos dos programas oficiales reflejan la política educativa y curricular que promueven diferentes formas de pensar a través de la resolución de problemas y la indagación, de manera conjunta como el "saber ser", se vuelve indispensable implementar procesos de supervisión y evaluación, debido a que las ideas expuestas en las mallas curriculares no necesariamente, están siendo implementadas en las aulas de secundaria o en los entornos educativos (Lentini y Villalobos, 2014; Espinoza y Zumbado, 2015; PREMCR, 2015; Meza, Agüero y Suárez, 2019).

Es preciso ofrecer una educación que satisfaga las cuatro características indicadas y producir aprendices expertos, pero es ineludible 
medir el avance de las ideas que orientan los procesos de enseñanza y aprendizaje propuestas por el MEP, pero sin olvidar los otros asuntos no tratados aquí: los aspectos administrativos y de infraestructura que complementan la educación como derecho humano, porque su ausencia entorpece la "disponibilidad".

Finalmente, se debe indicar que las ideas educativas costarricenses parecen estar en la dirección correcta y se debe invitar a los docentes a capacitarse y autocapacitarse sobre las temáticas y ejecutar los programas oficiales según su estrategia metodológica y su fundamentación, con el objetivo de conseguir los perfiles de salida esperados por la entidad ministerial, donde los aprendices expertos, podrán interpretar, analizar, justificar, establecer prioridades, formular y reformular ideas o propuestas, inferir, evaluar y argumentar sobre situaciones del contextos con una perspectiva integral (MEP, 2015).

\section{Referencias}

Bosman, A. y Schulze, S. (2018). Learning style preferences and Mathematics achievement of secondary school learners. South African Journal of Education, 38(1), 1-8. Recuperado de https://files. eric.ed.gov/fulltext/EJ1173186.pdf

Callejos, M. (2000). Educación matemática y ciudadanía: propuestas desde los Derechos Humanos. República Dominicana: Editorial Centro Cultural Poveda. Recuperado de https://www.centropoveda.org/IMG/pdf/matematicasDDHH.pdf

Calvo, C., Deulofeu, J., Jareño, J. y Morera, L. (2016). Aprender a enseñar matemáticas en la educación secundaria obligatoria. Madrid: Editorial Síntesis.

Chaves, E. (2016). La enseñanza de la Estadística y la Probabilidad, más allá de procedimientos y técnicas. Cuadernos de Investigación y Formación en Educación Matemática, 11(15), 21-31 Recuperado de https://revistas.ucr.ac.cr/index.php/cifem/article/ view/23880/24056

Espinoza, J. y Zumbado, M. (2015). Planes piloto en la implementación de nuevos Programas de Matemáticas. Cuadernos de Investigación y Formación en Educación Matemática, 10(13), 227-248. Recuperado de https://revistas.ucr.ac.cr/index.php/cifem/article/ view/19160/19222 
Lentini, V. y Villalobos, J. (2014). Condiciones en que se aplica la reforma curricular de matemáticas en colegios públicos diurnos, según los docentes. Ponencia preparada para el Quinto Informe del Estado de la Educación. San José: PEN.

Limas, M. y Ampudia, L. (2013). ¿La educación es un derecho humano o es un factor del desarrollo humano? Una aproximación a la reflexión situando los casos de Chile, España y México. Memorias del Foro Bienal Iberoamericano de Estudios del Desarrollo, Chile: RIED. Recuperado de https://www.estudiosregionales. org/app/download/6526488554/Educación.pdf?t=1358050596

Meza, G., Agüero, E. y Suárez, Z. (2019). Reforma de la educación matemática en Costa Rica: evaluación de avance de la implementación en la educación secundaria. Revista digital Matemática, Educación e Internet, 19(2), 1-26. Recuperado de https://doi. org/10.18845/rdmei.v19i2.4218

Ministerio de Educación Pública [MEP]. (2018a). Informe de implementación 2012-2017. San José, Costa Rica: Autor. Recuperado de https:/www.reformamatematica.net/wp-content/uploads/2019/04/ Informe-MEP_Reforma-Matematica-hasta-2017.pdf

Ministerio de Educación Pública [MEP]. (2018b). Diseño universal para el aprendizaje (DUA). Recuperado de http://www.drea.co. $\mathrm{cr} / \mathrm{sites} /$ default/files/Contenido/09.\%20Folleto\%20Dise\%C3\%B10\%20Univesal\%20del\%20Aprendizaje.pdf

Ministerio de Educación Pública [MEP]. (2017a). Política educativa. La persona: centro del proceso educativo y sujeto transformador de la sociedad. San José, Costa Rica: Autor. Recuperado de http://www.mep.go.cr/sites/default/files/page/adjuntos/politicaeducativa.pdf

Ministerio de Educación Pública [MEP]. (2017b). Programas de estudio de Ciencias. Primer y Segundo Ciclos de Educación General Básica. San José, Costa Rica: Autor. Recuperado de https://www. mep.go.cr/sites/default/files/programadeestudio/programas/ciencias1y2ciclo2018.pdf

Ministerio de Educación Pública [MEP]. (2017c). Programas de estudio de Ciencias. Tercer Ciclo de Educación General Básica. San José, Costa Rica: Autor. Recuperado de https://mep.go.cr/sites/ default/files/programadeestudio/programas/ciencias3ciclo.pdf 
Ministerio de Educación Pública [MEP]. (2017d). Programas de estudio de Física. Educación Diversificada. San José, Costa Rica: Autor. Recuperado de https://www.mep.go.cr/sites/default/files/ programadeestudio/programas/fisica2018.pdf

Ministerio de Educación Pública [MEP]. (2017e). Programas de estudio de Química. Educación diversificada. San José, Costa Rica: Autor. Recuperado de https://www.mep.go.cr/sites/default/files/ programadeestudio/programas/quimica2018.pdf

Ministerio de Educación Pública [MEP]. (2017f). Programas de estudio de Biología. Educación Diversificada. San José, Costa Rica: Autor. Recuperado de https://www.mep.go.cr/sites/default/files/ programadeestudio/programas/biologia2017.pdf

Ministerio de Educación Pública [MEP]. (2015). Educar para una nueva ciudadanía: fundamentación de la transformación curricular costarricense. Costa Rica: Autor. Recuperado de http://www.idp. mep.go.cr/sites/all/files/idp_mep_go_cr/publicaciones/7-2016_ educar_para_una_nueva_ciudadaniafinal.pdf

Ministerio de Educación Pública [MEP]. (2012). Programas de estudio de Matemáticas para la Educación General Básica y el Ciclo Diversificado. San José, Costa Rica: Autor. Recuperado de http:// www.mep.go.cr/sites/default/files/programadeestudio/programas/matematica.pdf

Muñoz, V. (2013). El derecho a la Educación: una mirada comparativa. Argentina, Chile, Uruguay y Finlandia. Santiago, Chile: OREAL/UNESCO. Recuperado de http://www.unesco.org/ new/fileadmin/MULTIMEDIA/FIELD/Santiago/images/Estudio-comparativo-UNESCO-vernor-munoz-espanol.pdf

National Council of Teachers of Mathematics [NCTM]. (2000). Resumen ejecutivo principios y estándares para la Educación Matemática (Claudia Matus, trad.). México: CIAEM. (Obra original publicada en 2000). Recuperado de http://www.cimm.ucr.ac.cr/ ciaem/archivos/RE_NCTM.pdf

OCDE (2017). Marco de evaluación y de análisis de PISA para el desarrollo: lectura, matemáticas y ciencias. Versión preliminar, París: OECD Publishing. Recuperado de https://www.oecd.org/ pisa/aboutpisa/ebook\%20-\%20PISA-D\%20Framework_PRELIMINARY\%20version_SPANISH.pdf 
Organización de Naciones Unidas [ONU]. (1948). Declaración Universal de Derechos Humanos. Recuperado de https://www.ohchr. org/EN/UDHR/Documents/UDHR_Translations/spn.pdf

Organización de Naciones Unidas [ONU]. (1999). Consejo económico y social. Recuperado de http://www.refworld.org/cgi-bin/texis/ vtx/rwmain/opendocpdf.pdf?reldoc $=y \&$ docid $=47 \mathrm{ebcc} 8 \mathrm{e} 2$

Organización de las Naciones Unidas para la Educación, la Ciencia y la Cultura [UNESCO]. (2015). Resultados del foro mundial sobre la educación 2015. Recuperado de https://unesdoc.unesco.org/ ark:/48223/pf0000234002_spa

Ossa-Cornejo, C., Palma-Luengo, M., Lagos-San Martín, N. y DíazLarenas, C. (2018). Evaluación del pensamiento crítico y científico en estudiantes de pedagogía de una universidad chilena. Revista Electrónica Educare, 22(2), 1-18. Recuperado de http:// dx.doi.org/10.15359/ree.22-2.12

Programa Estado de la Nación [PEN]. (2017). Sexto informe estado de la educación. Primera Edición. San José, Costa Rica: Servicios Gráficos, A. C. Recuperado de: https://www.estadonacion.or.cr/ educacion2017/assets/preliminares-capitulo-1-ee6-en-baja.pdf

Proyecto Reforma de la Educación Matemática en Costa Rica [PREMCR]. (2015). Integración de habilidades del currículo oficial de Matemáticas en la acción en el aula. Cuadernos de Investigación y Formación en Educación Matemática, 10(13), 227-248. Recuperado de: https://revistas.ucr.ac.cr/index.php/cifem/article/ view/19160

Riveros, F. (2017). Mujer y matemáticas. Educación para la equidad de género y los Derechos Humanos. Educación y Ciudad, 32, 93-102. Recuperado de https://dialnet.unirioja.es/descarga/articulo/6213565.pdf

Ruiz, A. (2018). Evaluación y pruebas naciones para un currículo de Matemáticas que enfatiza capacidades superiores. México: CIAEM.

Ruiz, A. (2014). La implementación de los programas oficiales de matemáticas. Quinto informe del Estado de la Educación. San José: PEN. Recuperado de https://estadonacion.or.cr/files/biblioteca virtual/educacion/005/Angel_Ruiz_La_implementacion_programas_matemt.pdf 
Ruiz, A. (2013). Reforma de la Educación Matemática en Costa Rica. Perspectiva de la praxis. Cuadernos de Investigación y Formación en Educación Matemática, 8 (Número especial), 1-111. Recuperado de: https://revistas.ucr.ac.cr/index.php/cifem/article/ view/11125

Ruiz, A. y Barrantes, H. (2015). Informe técnico sobre la implementación de los programas oficiales de Matemáticas. Cuadernos de Investigación y Formación en Educación Matemática, 10(13), 227-248. Recuperado de https://revistas.ucr.ac.cr/index.php/ cifem/article/view/19160/19222

Tamayo, O. y Orrego, M. (2005). Aportes de la naturaleza de la ciencia y del contenido pedagógico del conocimiento para el campo conceptual de la educación en ciencias, Revista Educación y Pedagogía, 17(43), 13-25.

Torres, R. (2005). Justicia educativa y justicia económica, 12 tesis para el cambio educativo. Bogota: Iarriccio Artes Gráficas. 
